\title{
Singularity-invariant Leg Rearrangements in Doubly-planar Stewart-Gough Platforms
}

\author{
Júlia Borràs, Federico Thomas, and Carme Torras \\ Institut de Robòtica i Informàtica Industrial (CSIC-UPC), Barcelona, Spain \\ E-mails: \{jborras, fthomas, ctorras\}@iri.upc.edu
}

\begin{abstract}
In general, rearranging the legs of a Stewart-Gough platform, i.e., changing the locations of its leg attachments, modifies the platform singularity locus in a rather unexpected way. Nevertheless, some leg rearrangements have been recently found to leave singularities invariant but, unfortunately, these rearrangements are only valid for Stewart-Gough platforms containing rigid components. In this work, the authors go a step further presenting singularity-invariant leg rearrangements that can be applied to any Stewart-Gough platform whose base and platform attachments are coplanar. The practical consequences of the presented theoretical results are illustrated with several examples including well-known architectures.
\end{abstract}

\section{INTRODUCTION}

Even when there is no known solution to a given mathematical problem, it is always possible to try to find the set of transformations to the problem that leave its solution invariant. Although this does not solve the problem itself, it provides a lot of insight into its nature. This way of thinking is at the root of the development of Group Theory and it is the one applied herein for the characterization of the singularity loci of Stewart-Gough platforms.

The Stewart-Gough platform is defined as a 6-DoF parallel mechanism with six identical SPS legs [14], [6]. It triggered the research on parallel manipulators, and it has remained one of the most widely studied because, despite its geometric simplicity, its analysis translates into challenging mathematical problems. One important part of this analysis corresponds to the characterization of its singularities.

The geometric and topological characterization of the singularity locus of a given Stewart-Gough platform in its sixdimensional configuration space is, in general, a huge task which has only been completely solved for some specializations -i.e., designs in which some spherical joints in the platform, the base, or both, coalesce to form multiple spherical joints [2], [1]. In this context, it seems reasonable to find leg rearrangements in a given Stewart-Gough platform that leave its singularity locus invariant for two main reasons:

(a) If the singularity locus of the platform at hand has already been characterized, it could be interesting to modify the location of its legs to optimize some other platform characteristics without altering such locus.

(b) If the singularity locus of the analyzed platform has not been characterized yet, it could be of interest to simplify the platform's geometry by changing the location of its legs, thus easing the task of obtaining this characterization.

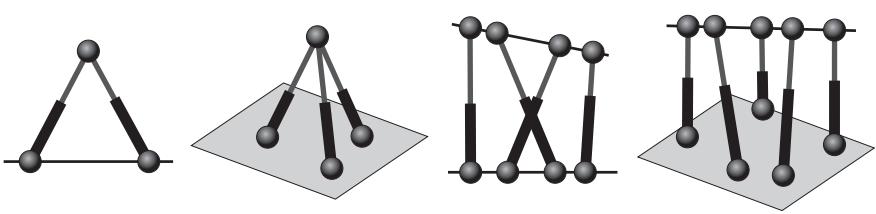

Fig. 1. The four possible rigid components involving linear geometric elements in Stewart-Gough platforms.

Let us suppose that we want to apply a singularity-invariant leg rearrangement limited to a subset of legs. Clearly, this is only possible if this subset of legs defines a rigid subassembly. Kong and Gosselin refer to these subassemblies as components [12]. The simplest component arises when two legs share an attachment. The result is called the Point-Line component. Similarly, the three other components involving linear geometric entities (points, lines and planes) are the Point-Plane, Line-Line and Line-Plane components (Fig.1). The singularity-invariant leg rearrangements for each of these four components have already been fully characterized [4], [3], [5]. In this paper, the authors go a step further presenting the rules for the leg rearrangements that can be applied to any Stewart-Gough platform whose base and platform attachments are coplanar (i.e., the equivalent to a Plane-Plane component).

We will show that, for a leg rearrangement to be singularityinvariant, it is necessary and sufficient that the linear actuators' velocities, before and after the rearrangement, are linearly related. By integrating this differential condition, the above statement can be reformulated as follows: a leg rearrangement is singularity-invariant if the squared leg lengths, before and after the rearrangement, are affinely related. It is important to realize that, if this condition is satisfied, a one-to-one correspondence between the elements of the platform forward kinematics solution sets, before and after the rearrangement, arises. Actually, the invariance in the singularities and the assembly modes of a parallel platform are two faces of the same coin. These ideas are closely related to those that made possible the development of kinematic substitutions [8]. They are general in the sense that they can be applied to any kind of mechanism, not only parallel platforms. We will also show that their application to well-studied platforms leads to interesting new results. For example, we will see that it is not necessary that a platform has collinear attachments to behave like a Griffis-Duffy type II manipulator.

This paper is organized as follows. In Section II, a nec- 
essary and sufficient condition that must be satisfied by any singularity-invariant leg rearrangement in a Stewart-Gough platform is presented. Then, the challenge becomes that of finding the geometric transformations that satisfy this condition. This is discussed in Sections III and IV for any doublyplanar Stewart-Gough platform. Section V presents some cases that exemplify the potentialities of the obtained transformation. Finally, Section VI summarizes the main results.

\section{GENERAL CONDITION FOR A LEG REARRANGEMENT TO BE SINGULARITY-INVARIANT}

In general, if we change the location of the leg attachments in a Stewart-Gough platform, its singularity locus is modified. However, it is shown below that the singularity locus remains invariant if, and only if, the squared lengths of the legs in their new location can be expressed in terms of those in the original location through an affine relation (for any arbitrary pose of the platform with respect to the base).

Let us consider a general Stewart-Gough platform, i.e., a 6-DoF parallel mechanism with six identical SPS legs [14], [6]. For this kind of platform, the linear actuators' velocities, $\dot{l}_{1}, \dot{l}_{2}, \ldots, \dot{l}_{6}$, can be expressed in terms of the platform velocity vector $(\mathbf{v}, \boldsymbol{\Omega})$ as follows:

$$
\operatorname{diag}\left(l_{1}, \ldots, l_{6}\right)\left(\begin{array}{c}
i_{1} \\
i_{2} \\
\vdots \\
i_{6}
\end{array}\right)=\mathbf{J}\left(\begin{array}{c}
\mathbf{v} \\
\boldsymbol{\Omega}
\end{array}\right),
$$

where $\mathbf{J}$ is the matrix of normalized Plücker coordinates of the six leg lines [14]. The parallel singularities of the platform are those configurations in which $\operatorname{det}(\mathbf{J})=0$ [13].

Now, let us change the location of the leg attachments so that the lengths of the legs in their new locations, say $d_{1}, d_{2}, \ldots, d_{6}$, are related to those of the original legs, $l_{1}, l_{2}, \ldots, l_{6}$, through the relation:

$$
\left(\begin{array}{c}
d_{1}^{2} \\
d_{2}^{2} \\
\vdots \\
d_{6}^{2}
\end{array}\right)=\mathbf{A}\left(\begin{array}{c}
l_{1}^{2} \\
l_{2}^{2} \\
\vdots \\
l_{6}^{2}
\end{array}\right)+\mathbf{b}
$$

where $\mathbf{A}$ and $\mathbf{b}$ are a constant matrix and a constant vector, respectively. Differentiating this equation with respect to time and substituting (1) in the result, we get

$$
\operatorname{diag}\left(d_{1}, \ldots, d_{6}\right)\left(\begin{array}{c}
\dot{d}_{1} \\
\dot{d}_{2} \\
\vdots \\
\dot{d}_{6}
\end{array}\right)=\mathbf{A J}\left(\begin{array}{c}
\mathbf{v} \\
\boldsymbol{\Omega}
\end{array}\right)
$$

Then, the singularities of the platform after the leg rearrangement leading to (2) are those configurations in which $\operatorname{det}(\mathbf{A J})=\operatorname{det}(\mathbf{A}) \operatorname{det}(\mathbf{J})=0$. If $\operatorname{det}(\mathbf{A}) \neq 0$, the leg rearrangement is said to be singularity-invariant. If $\operatorname{det}(\mathbf{A})=$ 0 , the rearrangement introduces an architectural singularity, i.e., the platform is always in a singularity irrespective of its leg lengths.

Since lengths are assumed to be positive magnitudes, equation (2) defines a one-to-one relationship between leg lengths before and after a singularity-invariant leg rearrangement. As a consequence, this kind of transformations leaves not only the singularities of the platform unaltered, but also the nature and number of its assembly modes.

\section{LEG REARRANGEMENTS}

It has been shown in the preceding section that any singularity-invariant leg rearrangement results in an affine relation between the leg lengths before and after the rearrangement. In this section this idea is exploited to define the singularity-invariant conditions for the doubly-planar general Stewart-Gough platform.

In a doubly-planar Stewart-Gough platform, the $i$-th leg joins a base attachment with coordinates $\mathbf{a}_{i}=\left(x_{i}, y_{i}, 0\right)^{T}$ with a platform attachment whose local coordinates are $\widehat{\mathbf{b}}_{i}=$ $\left(z_{i}, t_{i}, 0\right)^{T}$. Given the position $\mathbf{p}=\left(p_{x}, p_{y}, p_{z}\right)^{T}$ and the rotation

$$
\mathbf{R}=(\mathbf{i}, \mathbf{j}, \mathbf{k})=\left(\begin{array}{lll}
i_{x} & j_{x} & k_{x} \\
i_{y} & j_{y} & k_{y} \\
i_{z} & j_{z} & k_{z}
\end{array}\right)
$$

the coordinates of the platform attachments in the base reference frame are $\mathbf{b}_{i}=\mathbf{p}+\mathbf{R}\left(z_{i}, t_{i}, 0\right)^{T}$, for $i=1 \ldots, 6$.

Let $l_{i}$ be the length of the original $i$-th leg, then $l_{i}^{2}=\| \mathbf{b}_{i}-$ $\mathbf{a}_{i} \|^{2}$, for $i=1, \ldots, 6$.

We define a leg rearrangement of a single leg, i.e., the substitution of any of the legs by another one going from the base attachment located at $\mathbf{a}=(x, y, 0)^{T}$ to the platform attachment at $\mathbf{b}=\mathbf{p}+\mathbf{R}(z, t, 0)^{T}$. We will call it leg rearrangement towards $(x, y, z, t)$. Now we proceed to compute the length of that newly introduced leg $d^{2}=\|\mathbf{b}-\mathbf{a}\|^{2}$.

Subtracting from the expressions for $d^{2}$ and $l_{i}^{2}, i=1, \ldots, 6$, the equations $i_{x}^{2}+i_{y}^{2}+i_{z}^{2}=1$ and $j_{x}^{2}+j_{y}^{2}+j_{z}^{2}=1$, and using the relation $\mathbf{i} \cdot \mathbf{j}=i_{x} j_{x}+i_{y} j_{y}+i_{z} j_{z}=0$, quadratic terms in the rotation variables cancel out, yielding

$$
\begin{aligned}
& -\frac{1}{2}\left(p_{x}^{2}+p_{y}^{2}+p_{z}^{2}\right)-z_{i} u-t_{i} v+x_{i} p_{x}+y_{i} p_{y} \\
& \quad+x_{i} z_{i} i_{x}+y_{i} z_{i} i_{y}+x_{i} t_{i} j_{x}+y_{i} t_{i} j_{y}-k_{i}=0 \\
& \quad-\frac{1}{2}\left(p_{x}^{2}+p_{y}^{2}+p_{z}^{2}\right)-z u-t v+x p_{x}+y p_{y} \\
& \quad+x z i_{x}+y z i_{y}+x t j_{x}+y t j_{y}-k+d^{2} / 2=0
\end{aligned}
$$

where $u=\mathbf{p} \cdot \mathbf{i}, v=\mathbf{p} \cdot \mathbf{j}$, and the constant factors $k_{i}=$ $\frac{1}{2}\left(x_{i}^{2}+y_{i}^{2}+z_{i}^{2}+t_{i}^{2}-l_{i}^{2}\right)$ and $k=\frac{1}{2}\left(x^{2}+y^{2}+z^{2}+t^{2}\right)$.

If we subtract the first equation from the others, quadratic terms in $p_{x}, p_{y}$ and $p_{z}$ cancel too, yielding six linear equations in the 9 unknowns $p_{x}, p_{y}, u, v, i_{x}, i_{y}, j_{x}, j_{y}$ and $d^{2}$ [equation (7)]. Note that $k_{i}$ depends on $l_{i}$.

From the matrix $\mathbf{Q}$ of the system (7), let us call $\mathbf{Q}_{i j k}$ the square matrix obtained from $\mathbf{Q}$ after deleting columns $i, j$ and $k$, and $Q_{i j k}$ its determinant. The system can be solved if we 


$$
\underbrace{\left(\begin{array}{ccccccccc}
z_{1}-z_{2} & t_{1}-t_{2} & x_{2}-x_{1} & y_{2}-y_{1} & x_{2} z_{2}-x_{1} z_{1} & y_{2} z_{2}-y_{1} z_{1} & x_{2} t_{2}-x_{1} t_{1} & y_{2} t_{2}-y_{1} t_{1} & 0 \\
z_{1}-z_{3} & t_{1}-t_{3} & x_{3}-x_{1} & y_{3}-y_{1} & x_{3} z_{3}-x_{1} z_{1} & y_{3} z_{3}-y_{1} z_{1} & x_{3} t_{3}-x_{1} t_{1} & y_{3} t_{3}-y_{1} t_{1} & 0 \\
z_{1}-z_{4} & t_{1}-t_{4} & x_{4}-x_{1} & y_{4}-y_{1} & x_{4} z_{4}-x_{1} z_{1} & y_{4} z_{4}-y_{1} z_{1} & x_{4} t_{4}-x_{1} t_{1} & y_{4} t_{4}-y_{1} t_{1} & 0 \\
z_{1}-z_{5} & t_{1}-t_{5} & x_{5}-x_{1} & y_{5}-y_{1} & x_{5} z_{5}-x_{1} z_{1} & y_{5} z_{5}-y_{1} z_{1} & x_{5} t_{5}-x_{1} t_{1} & y_{5} t_{5}-y_{1} t_{1} & 0 \\
z_{1}-z_{6} & t_{1}-t_{6} & x_{6}-x_{1} & y_{6}-y_{1} & x_{6} z_{6}-x_{1} z_{1} & y_{6} z_{6}-y_{1} z_{1} & x_{6} t_{6}-x_{1} t_{1} & y_{6} t_{6}-y_{1} t_{1} & 0 \\
z_{1}-z & t_{1}-t & x-x_{1} & y-y_{1} & x z-x_{1} z_{1} & y z-y_{1} z_{1} & x t-x_{1} t_{1} & y t-y_{1} & \frac{1}{2}
\end{array}\right)}_{\mathbf{Q}}\left(\begin{array}{c}
u \\
v \\
p_{x} \\
p_{y} \\
i_{x} \\
i_{y} \\
j_{x} \\
j_{y} \\
d^{2}
\end{array}\right)=\left(\begin{array}{c}
k_{2}-k_{1} \\
k_{3}-k_{1} \\
k_{4}-k_{1} \\
k_{5}-k_{1} \\
k_{6}-k_{1} \\
k-k_{1}
\end{array}\right)
$$

take three unknowns as parameters, for example $u, v, p_{x}$. The resulting linear system is:

$\mathbf{Q}_{123}\left(\begin{array}{c}p_{y} \\ i_{x} \\ i_{y} \\ j_{x} \\ j_{y} \\ d^{2}\end{array}\right)=\left(\begin{array}{c}\left(z_{2}-z_{1}\right) u+\left(t_{2}-t_{1}\right) v-\left(x_{2}-x_{1}\right) p_{x}+k_{2}-k_{1} \\ \left(z_{3}-z_{1}\right) u+\left(t_{3}-t_{1}\right) v-\left(x_{3}-x_{1}\right) p_{x}+k_{3}-k_{1} \\ \left(z_{4}-z_{1}\right) u+\left(t_{4}-t_{1}\right) v-\left(x_{4}-x_{1}\right) p_{x}+k_{4}-k_{1} \\ \left(z_{5}-z_{1}\right) u+\left(t_{5}-t_{1}\right) v-\left(x_{5}-x_{1}\right) p_{x}+k_{5}-k_{1} \\ \left(z_{6}-z_{1}\right) u+\left(t_{6}-t_{1}\right) v-\left(x_{6}-x_{1}\right) p_{x}+k_{6}-k_{1} \\ \left(z-z_{1}\right) u+\left(t-t_{1}\right) v-\left(x-x_{1}\right) p_{x}+k-k_{1}\end{array}\right)$.

Solving the system for $d^{2}$ using Crammer's rule and then applying multi-linear properties of determinants to split the determinant of the resulting matrix into 4 determinants yields

$$
d^{2}=\frac{Q_{239} u+Q_{139} v+Q_{129} p_{x}+Q_{123}^{*}}{\frac{1}{2} Q_{123}},
$$

where $Q_{123}^{*}$ is the determinant of $\mathbf{Q}_{123}$ except for the last column that contains the elements $k_{i}-k_{1}$ for $i=2, \ldots, 6$ and $k-k_{1}$. As a result, if we impose $Q_{239}=Q_{139}=$ $Q_{129}=0$, then equation (8) becomes affine in $l_{1}^{2}, \ldots, l_{6}^{2}$. Indeed, expanding $Q_{123}^{*}$ leads to an expression of the form

$$
d^{2}=c_{1} l_{1}^{2}+c_{2} l_{2}^{2}+c_{3} l_{3}^{2}+c_{4} l_{4}^{2}+c_{5} l_{5}^{2}+c_{6} l_{6}^{2}+c_{0},
$$

where all coefficients are known constants. Then, following Section II, any leg rearrangement satisfying $Q_{239}=Q_{139}=$ $Q_{129}=0$ leaves singularities invariant.

In other words, if we substitute any leg by a new leg with base attachment located at $\mathbf{a}=(x, y, 0)^{T}$ and platform attachment at $\mathbf{b}=\mathbf{p}+\mathbf{R}(z, t, 0)^{T}$, the singularities will remain invariant as long as $(x, y, z, t)$ satisfies the system $Q_{239}=Q_{139}=Q_{129}=0$, where $Q_{i j k}$ can be simplified into a $7 \times 7$ determinant using simple row/column operations, yielding the following system:

$$
\begin{aligned}
Q_{239} & =\left|\begin{array}{lllllll}
y_{1} & x_{1} z_{1} & y_{1} z_{1} & x_{1} t_{1} & y_{1} t_{1} & z_{1} & 1 \\
y_{2} & x_{2} z_{2} & y_{2} z_{2} & x_{2} t_{2} & y_{2} t_{2} & z_{2} & 1 \\
y_{3} & x_{3} z_{3} & y_{3} z_{3} & x_{3} t_{3} & y_{3} t_{3} & z_{3} & 1 \\
y_{4} & x_{4} z_{4} & y_{4} z_{4} & x_{4} t_{4} & y_{4} t_{4} & z_{4} & 1 \\
y_{5} & x_{5} z_{5} & y_{5} z_{5} & x_{5} t_{5} & y_{5} t_{5} & z_{5} & 1 \\
y_{6} & x_{6} z_{6} & y_{6} z_{6} & x_{6} t_{6} & y_{6} t_{6} & z_{6} & 1 \\
y & x z & y z & x t & y t & z & 1
\end{array}\right|=0, \\
Q_{139} & =\left|\begin{array}{cccccccc}
y_{1} & x_{1} z_{1} & y_{1} z_{1} & x_{1} t_{1} & y_{1} t_{1} & t_{1} & 1 \\
y_{2} & x_{2} z_{2} & y_{2} z_{2} & x_{2} t_{2} & y_{2} t_{2} & t_{2} & 1 \\
y_{3} & x_{3} z_{3} & y_{3} z_{3} & x_{3} t_{3} & y_{3} t_{3} & t_{3} & 1 \\
y_{4} & x_{4} z_{4} & y_{4} z_{4} & x_{4} t_{4} & y_{4} t_{4} & t_{4} & 1 \\
y_{5} & x_{5} z_{5} & y_{5} z_{5} & x_{5} t_{5} & y_{5} t_{5} & t_{5} & 1 \\
y_{6} & x_{6} z_{6} & y_{6} z_{6} & x_{6} t_{6} & y_{6} t_{6} & t_{6} & 1 \\
y & x z & y z & x t & y t & t & 1
\end{array}\right|=0,
\end{aligned}
$$

$$
Q_{129}=\left|\begin{array}{ccccccc}
y_{1} & x_{1} z_{1} & y_{1} z_{1} & x_{1} t_{1} & y_{1} t_{1} & -x_{1} & 1 \\
y_{2} & x_{2} z_{2} & y_{2} z_{2} & x_{2} t_{2} & y_{2} t_{2} & -x_{2} & 1 \\
y_{3} & x_{3} z_{3} & y_{3} z_{3} & x_{3} t_{3} & y_{3} t_{3} & -x_{3} & 1 \\
y_{4} & x_{4} z_{4} & y_{4} z_{4} & x_{4} t_{4} & y_{4} t_{4} & -x_{4} & 1 \\
y_{5} & x_{5} z_{5} & y_{5} z_{5} & x_{5} t_{5} & y_{5} t_{5} & -x_{5} & 1 \\
y_{6} & x_{6} z_{6} & y_{6} z_{6} & x_{6} t_{6} & y_{6} t_{6} & -x_{6} & 1 \\
y & x z & y z & x t & y t & -x & 1
\end{array}\right|=0
$$

\section{A. Generalizing and simplifying the condition}

The above reasoning fails if $Q_{123}=0$ but, for a nonarchitecturally singular manipulator, a $6 \times 6$ matrix $\mathbf{Q}_{i j k}$ with non-zero determinant can always be found (otherwise, the 6 leg length equations would be linearly dependent). However, this may change the expression of the singularity-invariant leg rearrangement condition in equations (9)-(11).

To avoid such ambiguity, we can reformulate the condition in terms of rank deficiency of the matrix $\mathbf{Q}_{9}$ (that is, the matrix $\mathbf{Q}$ in equation (7) without the last column). The 5 first rows of $\mathbf{Q}_{9}$ are full rank for any non-architecturally singular manipulator. Furthermore, $\mathbf{Q}_{9}$ is rank defective if, and only if, all its submatrices have null determinant. However, it is only necessary to check 3 of its submatrix determinants. Thus, the condition in equations (9)-(11) is equivalent to the rank deficiency of $\mathbf{Q}_{9}$. The advantage of this formulation is that any set of 3 submatrices could be used instead of the three determinants in (9)-(11).

To simplify the notation, we consider the following simpler matrix:

$$
\mathbf{P}=\left(\begin{array}{ccccccccc}
-z_{1} & -t_{1} & x_{1} & y_{1} & x_{1} z_{1} & y_{1} z_{1} & x_{1} t_{1} & y_{1} t_{1} & 1 \\
-z_{2} & -t_{2} & x_{2} & y_{2} & x_{2} z_{2} & y_{2} z_{2} & x_{2} t_{2} & y_{2} t_{2} & 1 \\
-z_{3} & -t_{3} & x_{3} & y_{3} & x_{3} z_{3} & y_{3} z_{3} & x_{3} t_{3} & y_{3} t_{3} & 1 \\
-z_{4} & -t_{4} & x_{4} & y_{4} & x_{4} z_{4} & y_{4} z_{4} & x_{4} t_{4} & y_{4} t_{4} & 1 \\
-z_{5} & -t_{5} & x_{5} & y_{5} & x_{5} z_{5} & y_{5} z_{5} & x_{5} t_{5} & y_{5} t_{5} & 1 \\
-z_{6} & -t_{6} & x_{6} & y_{6} & x_{6} z_{6} & y_{6} z_{6} & x_{6} t_{6} & y_{6} t_{6} & 1 \\
-z & -t & x & y & x z & y z & x t & y t & 1
\end{array}\right) .
$$

Let us denote by $P_{i j}$ the determinant of the submatrix obtained from $\mathbf{P}$ after deleting columns $i$ and $j$, and $P_{i j k}$ the determinant of the submatrix formed by the first 6 rows of $\mathbf{P}$ after deleting columns $i, j$ and $k$.

Note that $P_{i j}=Q_{i j 9}$ for $i, j \neq 9$ and $P_{i j k}=\frac{1}{2} Q_{i j k}$ for $k \neq 9$. Using these relations it can be proved that $\mathbf{Q}_{9}$ is rank defective if, and only if, $\mathbf{P}$ is also rank defective. Thus, a much simpler condition can now be stated: a leg rearrangement 
towards $(x, y, z, t)$ leaves singularities invariant as long as the matrix $\mathbf{P}$ is rank defective.

One practical methodology to check rank deficiency is to apply Gaussian elimination on $\mathbf{P}$. The last row of the resulting matrix has 3 nonzero terms dependent on $x, y, z$ and $t$. The corresponding 3 equations are equivalent to the system $\{(9),(10),(11)\}$. Different equations arise depending on the order of the columns. For example, Gaussian elimination on matrix $\mathbf{P}$ as it appears in equation (12) leads to a matrix whose last row is

$$
\frac{1}{P_{789}}\left(\begin{array}{lllllllll}
0 & 0 & 0 & 0 & 0 & 0 & P_{89} & P_{79} & P_{78}
\end{array}\right) .
$$

Then, as long as $P_{789} \neq 0$, the singularity-invariant leg rearrangements are defined by the system

$$
\left\{P_{89}=0, P_{79}=0, P_{78}=0\right\} .
$$

Alternatively, if the matrix $\mathbf{P}$ columns are sorted as $[y, x z, y z, x t, y t, 1,-z,-t, x]$, then the corresponding system is $\{(9),(10),(11)\}$ and $P_{123}$ should be non-zero.

\section{GeOMETRIC InTERPRETATION OF THE CONDITION}

Note that any equation consisting of a submatrix determinant $P_{i j}$ equated to zero will be bilinear in the unknowns, but with different monomials. Let us consider the system of equations (13), which after cofactor expansion, leads to

$$
\left.\begin{array}{rl}
-P_{891} z+P_{892} t+P_{893} x & -P_{894} y+P_{895} x z \\
- & P_{896} y z+P_{897} x t=0 \\
-P_{791} z+P_{792} t+P_{793} x & -P_{794} y+P_{795} x z \\
- & P_{796} y z+P_{798} y t=0 \\
-P_{781} z+P_{782} t+P_{783} x & -P_{784} y+P_{785} x z \\
- & -P_{786} y z+P_{789}=0
\end{array}\right\}
$$

As the system is linear both in $(x, y)$ and in $(z, t)$, it can be rewritten in matrix form as

$$
\mathbf{S}_{b}\left(\begin{array}{c}
z \\
t \\
1
\end{array}\right)=\left(\begin{array}{l}
0 \\
0 \\
0
\end{array}\right)
$$

where $\mathbf{S}_{b}$ is the matrix

$$
\left(\begin{array}{ccc}
P_{895} x-P_{896} y-P_{891} & P_{892}+P_{897} x & P_{893} x-P_{894} y \\
P_{795} x-P_{796} y-P_{791} & P_{792}+P_{798} y & P_{793} x-P_{794} y \\
P_{785} x-P_{786} y-P_{781} & P_{782} & P_{783} x-P_{784} y+P_{789}
\end{array}\right),
$$

that only depends on $x$ and $y$ (the $b$ refers to base, as $x$ and $y$ are the coordinates of the base plane). The other way round, the system can also be written as

$$
\mathbf{S}_{p}\left(\begin{array}{l}
x \\
y \\
1
\end{array}\right)=\left(\begin{array}{l}
0 \\
0 \\
0
\end{array}\right)
$$

where now matrix $\mathbf{S}_{p}$ is

$$
\left(\begin{array}{ccc}
P_{893}+P_{895} z+P_{897} t & -P_{894}-P_{896} z & P_{892} t-P_{891} z \\
P_{793}+P_{795} z & P_{798} t-P_{794}-P_{796} z & P_{792} t-P_{791} z \\
P_{783}+P_{785} z & -P_{784}-P_{786} z & P_{782} t-P_{781} z+P_{789}
\end{array}\right),
$$

that only depends on $z$ and $t$ (and $p$ refers to platform, as $z$ and $t$ are the coordinates of the platform plane).

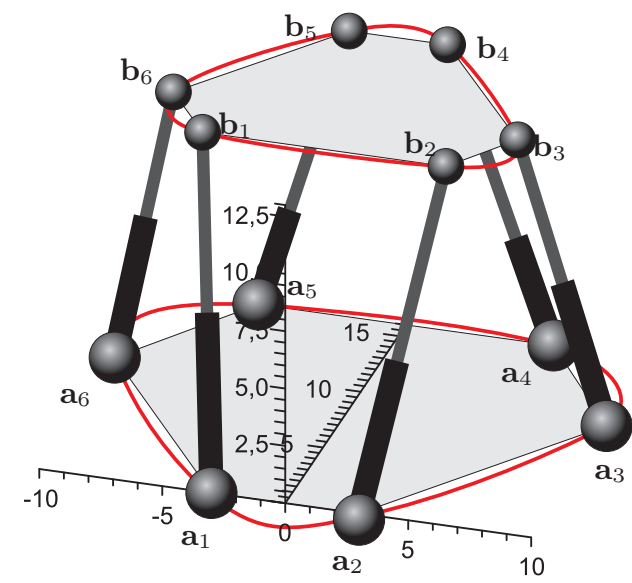

Fig. 2. Scheme of the platform described in Table I.

From equation (15) it is clear that the system has a solution on $(z, t)$ only for those $(x, y)$ that satisfy $\operatorname{det}\left(\mathbf{S}_{b}\right)=0$, and this solution is unique (assuming that the matrix $\mathbf{S}_{b}$ has rank 2). In the same way, there exists a solution on $(x, y)$ only for those $(z, t)$ that make $\operatorname{det}\left(\mathbf{S}_{p}\right)=0$. Both determinants define cubic curves on the base and platform planes, respectively. In other words, the system (14) defines a one-to-one correspondence between points on the two cubic curves. However, the correspondence may be not one-to-one for singular points on the cubics, as will be seen in the example of Section V-B.

Depending on the geometric placement of the attachments, these curves can be generic curves of degree 3 , or a line and a conic, or even 3 lines crossing 2 by 2 . This will be exemplified in the following section.

\section{EXAMPLES}

\section{A. Classic Stewart-Gough platform}

In [9] Husty et al. analyzed the classic Stewart-Gough platform, searching where additional legs could be placed without changing the forward kinematics solution, to obtain a redundant manipulator. The same example is analyzed here. The local coordinates of the attachments are listed in Table I.

TABLE I

COORDinATES OF THE ATTACHMENTS $\mathbf{a}_{i}=\left(x_{i}, y_{i}, 0\right)$ AND $\mathbf{b}_{i}=\mathbf{p}+\mathbf{R}\left(z_{i}, t_{i}, 0\right)^{T}$ FOR THE ANALYZED ROBOT

\begin{tabular}{|c|c|c|c|c|}
\hline$i$ & $x_{i}$ & $y_{i}$ & $z_{i}$ & $t_{i}$ \\
\hline \hline 1 & -3 & 0 & -5 & 0 \\
\hline 2 & 3 & 0 & 5 & 0 \\
\hline 3 & 10 & 10 & 7 & 3 \\
\hline 4 & 6 & 16 & 2 & 10 \\
\hline 5 & -6 & 16 & -2 & 10 \\
\hline 6 & -10 & 10 & -7 & 3 \\
\hline
\end{tabular}

After substituting the corresponding numerical values, the 

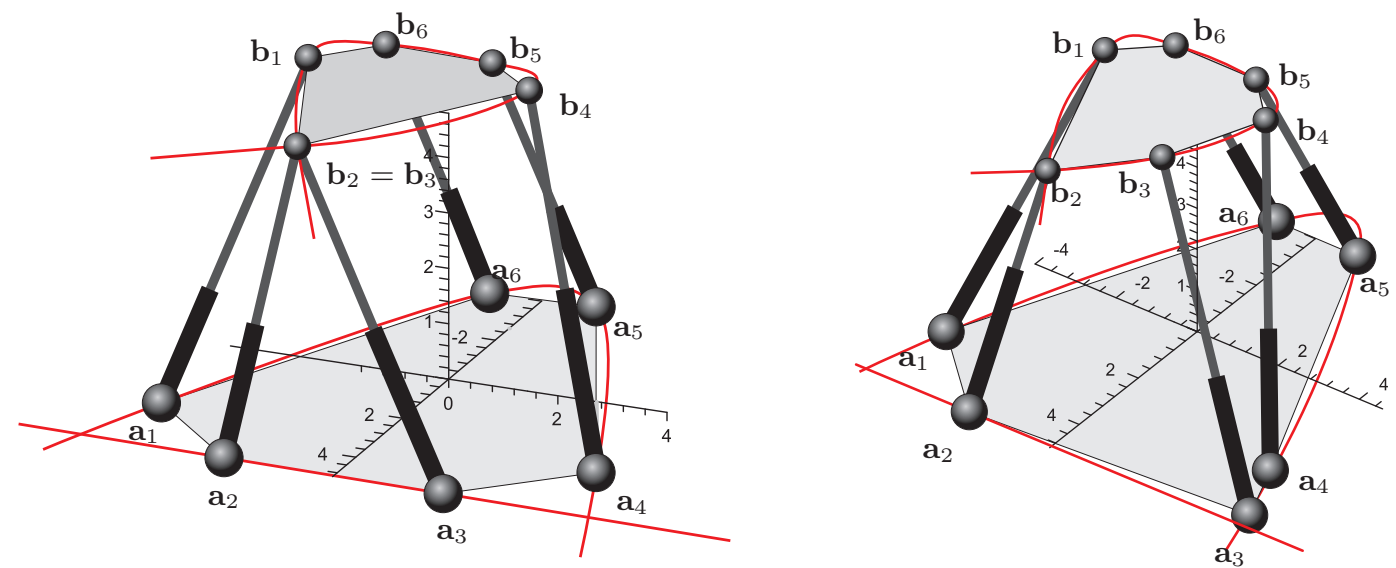

Fig. 3. Scheme of the platform described in Table II (left), and the equivalent platform after moving the 3rd leg (right).

system of equations (14) results in:

$$
\left.\begin{array}{rl}
2430 z-4050 x+255 y z+188 x t & =0 \\
-280 t+45 y+13 y t=0 \\
-70 t+43 y-4 x z+60=0
\end{array}\right\}
$$

and, thus, matrices $\mathbf{S}_{b}$ and $\mathbf{S}_{p}$ are:

$$
\begin{aligned}
\mathbf{S}_{b} & =\left(\begin{array}{ccc}
2430+255 y & 188 x & -4050 x \\
0 & 13 y-280 & 45 y \\
-4 x & -70 & 60+43 y
\end{array}\right), \\
\mathbf{S}_{p} & =\left(\begin{array}{ccc}
188 t-4050 & 255 z & 2430 z \\
0 & 13 t+45 & -280 t \\
-4 z & 43 & 60-70 t
\end{array}\right),
\end{aligned}
$$

whose determinants equated to zero give the two cubic curves

$$
\begin{aligned}
& -16296 x^{2} y+9503 y^{3}+302400 x^{2} \\
& \quad-47312 y^{2}-1599420 y-2721600=0 \\
& 20598 z^{2} t-8554 t^{3}+21870 z^{2} \\
& \quad+275173 t^{2}-1932795 t-546750=0
\end{aligned}
$$

plotted in Fig. 4. The cubic in the base coincides with the one appearing in [9], whereas the cubic in the platform is not given explicitly there.

By definition, all attachment coordinates are solutions of the system, and therefore belong to the curves, as shown in Fig.4.

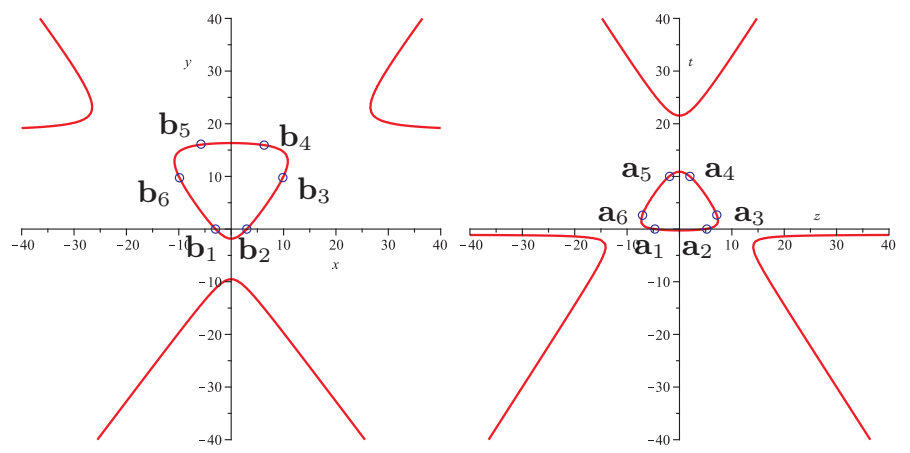

Fig. 4. The base and the platform curves defined in (17).
In [9], the authors propose to add additional legs to obtain redundant manipulators. Instead, here we propose to substitute any leg by another leg satisfying the one-to-one correspondence between the base and platform cubics defined by (17). The singularity locus will remain unchanged, and other performance indices can be improved, such as stiffness, or maneuverability, or the workspace can be enlarged by reducing the risk of leg collisions.

\section{B. Degenerate cubic curves}

Interesting cases appear when one or both of the curves are degenerate. Consider the example given in Table II, where two of the attachments on the platform are made coincident, $\mathbf{b}_{2}=\mathbf{b}_{3}$ [Fig. 3-(left)]. The two legs sharing an endpoint form a Point-Line component, and it was proved in [4] that the base attachments $\mathbf{a}_{2}$ and $\mathbf{a}_{3}$ can be rearranged on any point on the line $\mathbf{a}_{2} \mathbf{a}_{3}$ without modifying the singularity locus.

TABLE II

COORDinATES OF THE ATTACHMENTS $\mathbf{a}_{i}=\left(x_{i}, y_{i}, 0\right)$ AND $\mathbf{b}_{i}=\mathbf{p}+\mathbf{R}\left(z_{i}, t_{i}, 0\right)^{T}$ FOR THE ANALYZED ROBOT

\begin{tabular}{|c|c|c|c|c|}
\hline$i$ & $x_{i}$ & $y_{i}$ & $z_{i}$ & $t_{i}$ \\
\hline \hline 1 & 3 & -4 & -2 & -2 \\
\hline 2 & 5 & -2 & 2 & $-1 / 2$ \\
\hline 3 & 5 & 2 & 2 & $-1 / 2$ \\
\hline 4 & 3 & 4 & -2 & 2 \\
\hline 5 & -4 & 1 & -3 & 1 \\
\hline 6 & -4 & -1 & -3 & -1 \\
\hline
\end{tabular}

After applying Gaussian elimination to the matrix $\mathbf{P}$ given in equation (12) corresponding to this example, the following equations are obtained:

$$
\left.\begin{array}{r}
372 z+18988 t+1302 x-5656 y+527 x z \\
+2828 y z+1212 x t=0 \\
5172 z+808 t-2502 x+404 y+257 x z \\
+404 y z+2424 y t=0 \\
74 z-44 x-13 x z+202=0
\end{array}\right\}
$$




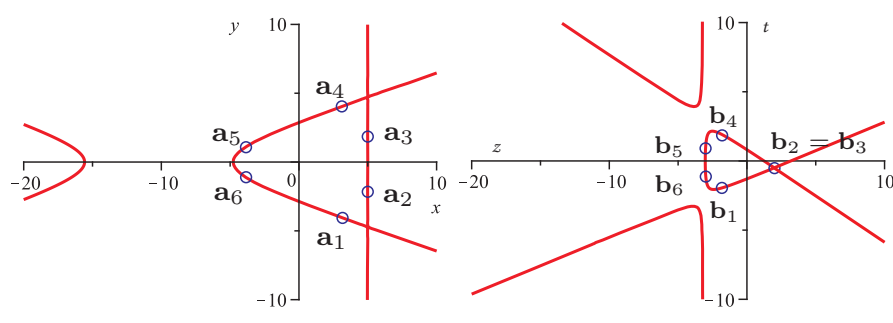

Fig. 5. The base and the platform curves defined in (19).

and the corresponding cubic curves are defined by

$$
\begin{aligned}
& 1713768(x-5) \\
& \left(31 x^{2}-280 y^{2}+631 x+2308\right)=0, \\
& 1713768\left(-132 z^{3}+124 z^{2} t+476 z t^{2}+191 z^{2}\right. \\
& \left.+620 z t+1528 t^{2}+1259 z+744 t-1606\right)=0 .
\end{aligned}
$$

In other words, the cubic curve in the base factorizes into a conic (an hyperbola) and a line, while the platform curve remains a cubic, but with a singular point (called node) on the vertex of the Point-Line component, $\mathbf{b}_{2}=(2,-1 / 2)$. Fig. 5 shows the plot of these two curves and the corresponding location of the attachments.

The correspondence between the base line and the platform cubic curve can be derived by solving system (18) for any point on the base line $\mathbf{a}_{2} \mathbf{a}_{3}$ (i.e., $x=5$ ), leading to

$$
\left.\begin{array}{rl}
3007 z+25048 t+6510-5656 y+2828 y z & =0 \\
+6457 z+808 t-12510+404 y+404 y z+2424 y t & =0 \\
9 z-18 & =0
\end{array}\right\}
$$

From the last equation, $z=2$. Substituting this value into the first two equations and factorizing the result yields

$$
\begin{aligned}
12524(1+2 t) & =0, \\
404(1+3 y)(1+2 t) & =0 .
\end{aligned}
$$

The solution of the system is $\{x=5, y=y, z=2, t=$ $-1 / 2\}$, that is, any point on the line $x=5$ corresponds to the vertex $\mathbf{b}_{2}=\mathbf{b}_{3}$ in accordance with previous results [4].

For the rest of the points on the cubic curve, the correspondence can be written in terms of a single parameter $z$. Given a point on the platform cubic

$$
\left(z, \frac{-31 z^{2}-155 z-186 \pm \sqrt{\Delta}}{2(119 z+382)}\right),
$$

the corresponding point on the base hyperbola is

$$
\begin{aligned}
& \left(\frac{2(37 z+101)}{44+13 z},\right. \\
& \left.\frac{3(z-2)\left(16669 z^{2}+103981 z+162022\right) \mp(26 z+88) \sqrt{\Delta}}{4(2-z)(44+13 z)^{2} \pm 2(39 z+132) \sqrt{\Delta}}\right),
\end{aligned}
$$

where the discriminant $\Delta=\left(16669 z^{2}+103981 z+\right.$ $162022)(z-2)^{2}$ determines whether points are real or complex. Real points on the platform always correspond to real points on the base, and vice versa. Observe that, on the singular point $z=2,(20)$ is undefined. However, terms $(z-2)$ can be simplified and then the resulting point gives the intersection between the line and the hyperbola. In other words, this parametrization represents the one-to-one correspondence between points on the platform cubic and the base hyperbola, except for the singular platform point $(2,-1 / 2)$, a double point that corresponds to two points on the hyperbola (the two intersections of the line with the conic).

To avoid multiple spherical joints, here the Point-Line component can be split by substituting any of its legs by another leg going from the conic to the base cubic. For example, take the point on the platform cubic given by $z=0$ and $t=\frac{-93+\sqrt{162022}}{382}$, and solve system (18) after evaluating it on this point, or equivalently, evaluate expression (20) for $z=0$. The result is:

$$
x=\frac{101}{22} \text { and } y=\frac{243033-44 \sqrt{162022}}{-3872+132 \sqrt{162022}} .
$$

Hence, we can substitute the 3rd leg by a new leg going from the base point $\left(\frac{101}{22}, \frac{243033-44 \sqrt{162022}}{-3872+132 \sqrt{162022}}, 0\right)$ to the platform attachment with local coordinates $\left(0, \frac{-93+\sqrt{162022}}{382}, 0\right)$ and the resulting Jacobian is the same as before, multiplied by a constant $\frac{15990+93 \sqrt{162022}}{67232}=0.794$, and with no coincident attachments (Fig. 3-right).

\section{Griffis-Duffy platforms}

In 1993, Griffis and Duffy patented two manipulators named thereafter Griffis-Duffy type I and II platforms [7]. Both platforms have their attachments distributed on triangles, three attachments on the vertices and three on the midpoints of the edges. Type I platforms are formed by joining the attachments on the midpoints on the base to the vertices on the platform, and the vertices on the base to midpoints on the platform (Fig. 6-left). Type II join midpoints to midpoints and vertices to vertices (Fig. 7-left).

A type I Griffis-Duffy platform is shown to be singularity equivalent to the octahedral manipulator [4]. In [11], type II Griffis-Duffy manipulators are shown to be always nonarchitecturally singular.

Consider the two examples specified in Table III, where the same triangles define two manipulators of type I and type II, respectively.

\section{TABLE III}

CoORdinates of THE ATTACHMENTS $\mathbf{a}_{i}=\left(x_{i}, y_{i}, 0\right)$ AND $\mathbf{b}_{i}=\mathbf{p}+\mathbf{R}\left(z_{i}, t_{i}, 0\right)^{T}$ FOR THE ANALYZED ROBOT

\begin{tabular}{|c|c|c|}
\hline$i$ & $z_{i}$ & $t_{i}$ \\
\hline \hline 1 & 1 & 0 \\
\hline 2 & $1 / 2$ & 0 \\
\hline 3 & -1 & 0 \\
\hline 4 & $-1 / 2$ & $\sqrt{3} / 2$ \\
\hline 5 & 0 & $\sqrt{3}$ \\
\hline 6 & $1 / 2$ & $\sqrt{3} / 2$ \\
\hline
\end{tabular}

\begin{tabular}{|c|c|c|c|}
\hline$i$ & $i$ & & \\
Type I & Type II & $x_{i}$ & $y_{i}$ \\
\hline \hline 2 & 1 & 2 & 0 \\
\hline 3 & 2 & $2 / 3$ & 0 \\
\hline 4 & 3 & -2 & 0 \\
\hline 5 & 4 & $-2 / 3$ & $(4 / 3) \sqrt{3}$ \\
\hline 6 & 5 & 0 & $2 \sqrt{3}$ \\
\hline 1 & 6 & 1 & $\sqrt{3}$ \\
\hline
\end{tabular}



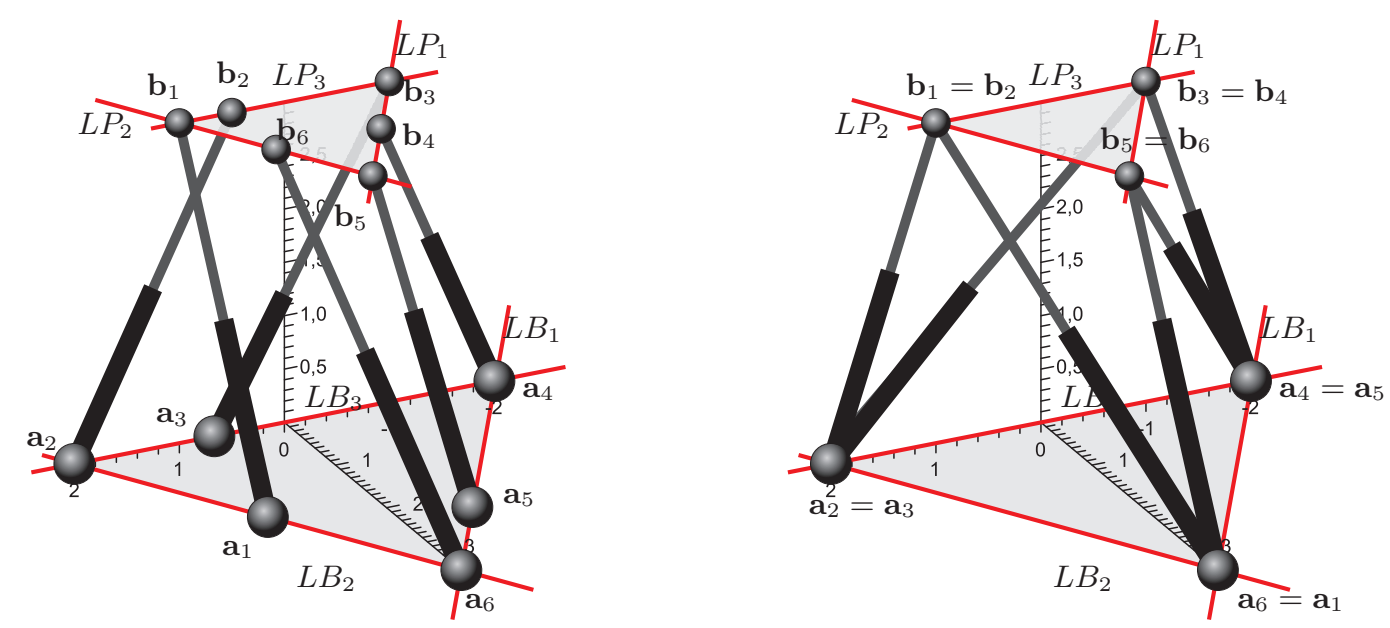

Fig. 6. Scheme of the platform described in Table III of a Griffis-Duffy type I platform (left), and its equivalent octahedral manipulator after applying a leg rearrangement (right).

The computation of the base and platform curves factorizes into the 3 same lines for both type I and type II platforms:

$$
\begin{aligned}
& \underbrace{(\sqrt{3} z-t+\sqrt{3})}_{L P_{1}} \underbrace{(\sqrt{3} z+t-\sqrt{3})}_{L P_{2}} \underbrace{t}_{L P_{3}}=0, \\
& \underbrace{(-3 x+\sqrt{3} y-6)}_{L B_{1}} \underbrace{(3 x+\sqrt{3} y-6)}_{L B_{2}} \underbrace{y}_{L B_{3}}=0 ;
\end{aligned}
$$

but the system obtained by applying Gaussian elimination on matrix $\mathbf{P}$ results in different equations. The system corresponding to the type I platform is:

$$
\left.\begin{array}{rl}
2 t-y+y z+x t & =0 \\
(\sqrt{3} z+t-\sqrt{3}) y & =0 \\
-2 \sqrt{3} z+4 t+\sqrt{3} x-y+\sqrt{3} x z+3 y z-2 \sqrt{3} & =0
\end{array}\right\}
$$

whereas for the type II platform is:

$$
\left.\begin{array}{r}
2 t-y+x t-y z=0 \\
3 \sqrt{3} y-8 \sqrt{3} t+\sqrt{3} y z+y t=0 \\
10 \sqrt{3} z-16 t-5 \sqrt{3} x+9 y+\sqrt{3} x z+y z-2 \sqrt{3}=0
\end{array}\right\}
$$

The resolution of these systems gives correspondences between base and platform attachments that leave the singularities invariant.

For the type I platform, the correspondence is between points and lines (in accordance with results in [4]), that is, to each vertex of the base (platform) triangle corresponds a line on the platform (base) triangle, in the same way as in the preceding section the vertex of the Point-Line component was in correspondence with a line on the base. Thus, by moving the six midpoint attachments along their supporting lines, the manipulator can be rearranged into the equivalent octahedral manipulator depicted in Fig. 6-right (a result concordant with that in [4]).

On the other hand, for the type II platform, there is a one-toone correspondence between points on line $L P_{i}$ and $L B_{i}$, for $i=1,2,3$. That is, the same geometrical elements determine the invariance of the singularity locus, but in the first case the correspondence is between points and lines and in the second case it is point-to-point between lines.

The legs of the type II manipulator can be rearranged following the correspondence $L B_{i} \leftrightarrow L P_{i}$. But some rearrangements must be avoided, for example, placing four legs in the same line-line correspondence leads to an architecturally singular manipulator (as it contains a Line-Line component in projective correspondence $[4,10])$.

An interesting rearrangement consists in removing all collinearities from the type II manipulator. As a result, an equivalent platform such as that shown in Fig. 7-right is obtained.

To remove collinearities, all legs from vertex to vertex need to be rearranged. The 1st leg can be placed going from a point on $L B_{2}$ to the corresponding point on $L P_{2}$. In other words, take a point on the line $L B_{2}$, substitute the values on system (21) and the solution gives a point on the line $L P_{2}$ :

$$
\underbrace{\begin{array}{c}
x=1 / 2 \\
y=(3 / 2) \sqrt{3}
\end{array}}_{\text {on } L B_{2}} \quad \begin{gathered}
\text { Substitute on }(21) \\
\text { and solve }
\end{gathered} \underbrace{\begin{array}{c}
z=1 / 4 \\
t=(3 / 4) \sqrt{3}
\end{array}}_{\text {on } L P_{2}}
$$

The same can be done to substitute the 3rd leg by a leg going from $L B_{3}$ to $L P_{3}$ :

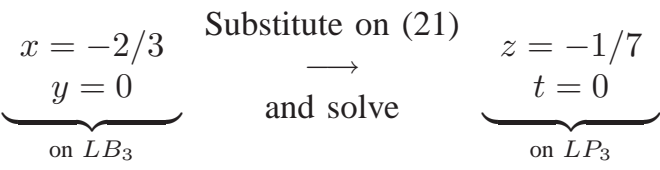

and finally, the 5th leg is substituted by a leg going from a point on $L B_{1}$ to a point on $L P_{1}$

$$
\underbrace{\begin{array}{cc}
x=-3 / 2 \\
y=(1 / 2) \sqrt{3}
\end{array}}_{\text {on } L B_{1}} \begin{gathered}
\text { Substitute on }(21) \\
\text { and solve }
\end{gathered} \underbrace{\begin{array}{c}
z=-6 / 7 \\
t=(1 / 7) \sqrt{3}
\end{array}}_{\text {on } L P_{1}}
$$



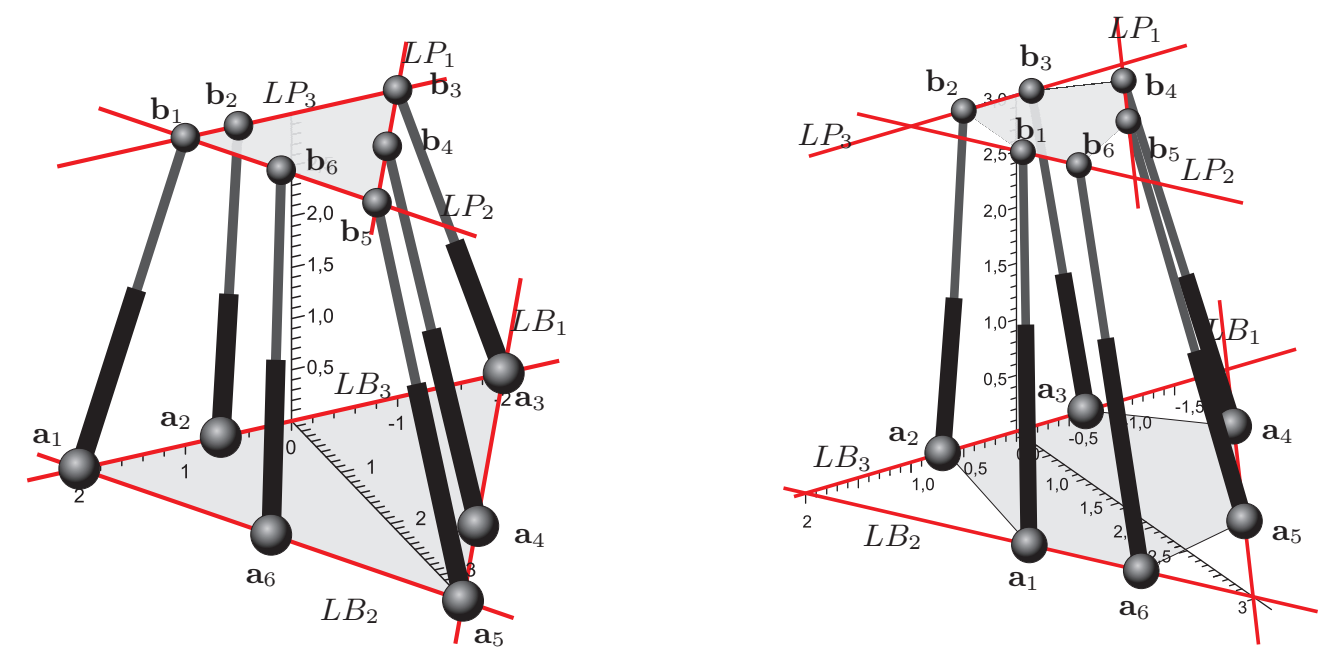

Fig. 7. Scheme of the platforms described in Table III of a Griffis-Duffy type II platform (left), and its equivalent platform after removing all collinearities (right).

The resulting manipulator depicted in Fig. 7-right is equivalent to the one in Fig.7-left, as regards to both its kinematics and its singularity locus.

In conclusion, we have seen that it is not necessary that a platform has collinear attachments to behave like a GriffisDuffy type II manipulator, and what is more important, we have found a general doubly-planar Stewart-Gough platform equivalent to a Griffis-Duffy type II manipulator.

\section{CONCLUSIONS AND FUTURE WORK}

In this work we have started to analyze the singularityinvariant leg rearrangements that can be applied to StewartGough platforms with coplanar attachments on the base and on the platform. It has been shown that the resulting attachments need to obey a one-to-one correspondence between two cubic curves, one on the base and another on the platform. The presented approach generalizes previous results.

Many promising possibilities open up for future research. There exist 15 different types of non-degenerate cubic curves, so we can classify all Stewart-Gough platforms according to the type of cubic curves they generate. Furthermore, many interesting cases can be studied that have degenerate cubic curves. In addition, efficient parameterizations of cubic curves available in literature would allow us to define the attachments using less parameters, thus simplifying the analysis.

On the other hand, we are working on the relation between the coefficients of the Jacobian polynomial and the minors of matrix $\mathbf{P}$ in equation (12), similarly as it was done in previous works for particular cases [3]. Based on the analysis of the $\mathbf{P}$ matrix, the number of assembly modes of the general StewartGough platform can be lowered by imposing simple relations between the attachments, as it was done in [5].

The usefulness of the proposed method has been shown with three examples. The derived leg rearrangements permit reducing the number of multiple spherical joints as well as devising reconfigurable robots that maintain the same singularity locus at each reconfiguration state.

\section{ACKNOWLEDGMENT}

This work has been partially supported by the Generalitat de Catalunya through the VALTEC program, cofinanced by FEDER funds, and the Spanish Ministry of Science and Innovation, under the I+D project DPI2007-60858.

\section{REFERENCES}

[1] M. Alberich-Carramiñana, M. Garolera, F. Thomas, and C. Torras, "Partially-flagged parallel manipulators: Singularity charting and avoidance," IEEE Transactions on Robotics, vol. 25, no. 4, pp. 771-784, 2009.

[2] M. Alberich-Carramiñana, F. Thomas, and C. Torras, "Flagged parallel manipulators," IEEE Transactions on Robotics, vol. 23, no. 5, pp. 10131023, 2007.

[3] J. Borràs and F. Thomas, "Kinematics of the line-plane subassembly in Stewart platforms," in IEEE International Conference on Robotics and Automation, 2009, pp. 4094-4099.

[4] J. Borràs, F. Thomas, and C. Torras, "On $\Delta$-transforms," IEEE Transactions on Robotics, vol. 25, no. 6, pp. 1225-1236, 2009.

[5] — , "A family of quadratically-solvable 5-UPS parallel robots," in IEEE International Conference on Robotics and Automation, 2010, pp. 4703-4708.

[6] B. Dasguptaa and T. Mruthyunjayab, "The Stewart platform manipulator: a review," Mechanism and Machine Theory, vol. 35, pp. 15-40, 2000.

[7] M. Griffis and J. Duffy, "Method and apparatus for controlling geometrically simple parallel mechanisms with distinctive connections," US Patent 5,179,525, 1993

[8] K. Hunt, Kinematic Geometry of Mechanisms. Oxford University Press, 1978 , p. 323.

[9] M. Husty, S. Mielczarek, and M. Hiller, "A redundant spatial StewartGough platform with a maximal forward kinematics solution set," in International Symposium on Advances in Robot Kinematics, 2002, pp. $147-154$.

[10] M. Husty and A. Karger, "Architecture singular parallel manipulators and their self-motions," in International Symposium on Advances in Robot Kinematics, 2000, pp. 355-364.

[11] — , "Self-motions of Griffis-Duffy type parallel manipulators," in IEEE International Conference on Robotics and Automation, 2000, pp. $7-12$.

[12] X. Kong and C. Gosselin, "Classification of 6-SPS parallel manipulators according to their components," in Proc. of ASME Design Engineering Technical Conferences, 2000, pp. DETC2000/MECH-14 105.

[13] O. Ma and J. Angeles, "Architecture singularities of platform manipulators," in IEEE International Conference on Robotics and Automation, vol. 2, 1991, pp. 1542-1547.

[14] J.-P. Merlet, Parallel Robots. Springer, 2000. 